\title{
Design of Batch Pressing Mechanism for Bedded Standard Samples
}

\author{
Jianguang Li, Yongyan Wang, Nan Qin, Huihua Zhu, Yanchun Wang \\ College of Electromechanical Engineering, Qingdao University of Science and Technology, Qingdao 266061, China
}

\begin{abstract}
In stability analysis of rock soil engineering, before establishing similar physical simulation model, many match experiments on similar material are required, the current standard sample forming processes are difficult to satisfy the uniformity and compactness in the same sample set often, and have obvious defects in the molding process, molding efficiency and bedding processing. A pressing mechanism consists of adjustable concave-convex composite mould, multi-function trough, drive screw and framework is designed, the mould is pressed nto material to fill directly. Each layered material, dip angle, thickness and position can be controlled, forming number for each lot is adjustable, uniform of the same lot is good, all parts are detachable, operation is also simple. This mechanism is suitable for small batch forming, and has obvious advantages in bedding controlling, forming efficiency, forming quality and operation convenience, which can work well for match experiments before large-scale physical similar simulation experiment, geotechnical experiment, composite rock mass experiment, also can be used to make micro physical model with engineering structure.
\end{abstract}

\section{Introduction}

In some design for large engineering, such as slope, caverns, storage and so on, stability analysis usually are done to forecast the possible deformation and damage of rock mass in engineering. Common stability analyses include physical model similar simulation and numerical simulation. In the physical model, large-scale geomechanical model is always made, according to the geological conditions, rock layers and geometrical size of current engineering, model materials for similar materials follow a similar ratio and proportion, which is related with the rock mechanical properties, engineering scale in actual geological environment. Due to discrete and uncertainty of underground rock mass, in each new project, the geomechanical model need a lot of preparation of similar material standard samples and conventional mechanical test. Usually, the test goal is getting the elastic modulus, compression strength and other parameters of similar materials at a certain similar ratio, and it is required a series of sample group with sufficient number and consistent performance, to ensure the reliability of data. In practice, different experimental project require different sample number, which often are done manually or by simple mold, and can meet the requirement of uniformity partly. For example, Ma zhenguo [1] et al make sample by pressing mechanism and matching die (similar method is widely used, most of them is operated manually, the pressing force is small and the forming number is small, see Fig. 1), but there are obvious disadvantages in forming efficiency and quality.
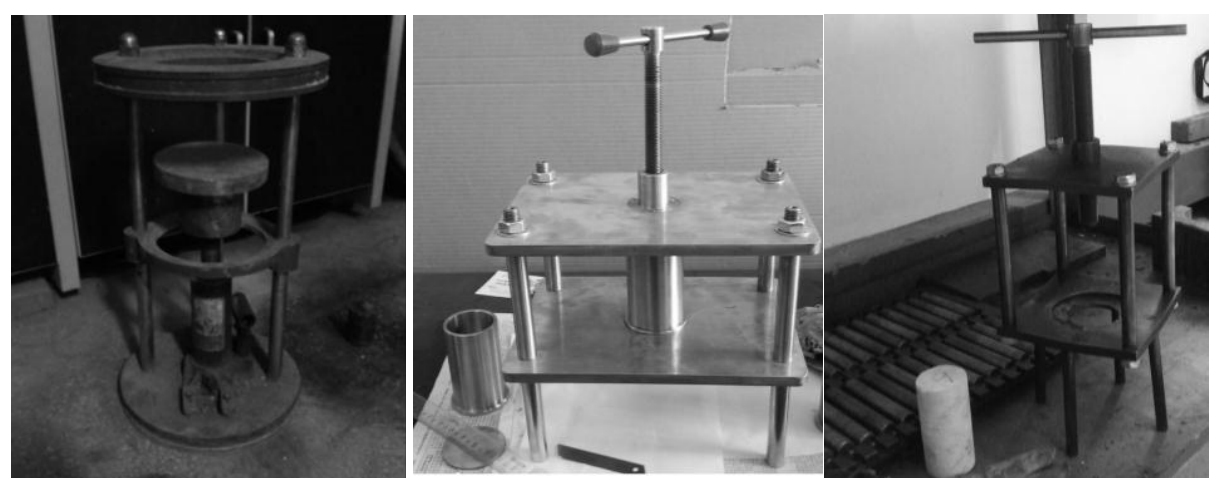

Figure 1. Common standard sample forming mechanism. 
If the sample contain bedding and have special requirements for angle and materials, it is difficult to ensure the consistency of the same batch of samples by making manually, and depend more on the special machinery and tooling. In essence, layered rock mass is composed by different material, thickness, angle, adhesion.etc, the production process or equipments, which can achieve any combination of above variables, all belong to reasonable forming process. Different researchers use different ideas and designs. Jiang de-yi et al [2] aimed to get the approximation of original rock mass, with the help of general press machine, pressed salt rock powder and interlayer material powder into cylindrical molding mould orderly, and obtained salt rock standard sample with horizontal interlayer; Yong ming tien [3] designed a set of equipment to make standard sample with any layer characteristic, by mixture, adsorption layer by layer, concentration pressure, cutting and drilling etc. Zhang gui-min [4] made further improvement based on Yong ming tien, he designed a set of thin layer adsorption, translation and cutting device, by which, the bedded standard sample with different angle and thickness ratio could be obtained.

The above designs have practicability to a certain degree, but there are also some disadvantages. Based on the comprehensive investigation on the previous research, author design a set of simple, efficient pressing mechanism, by which, standard sample with bedding, uniform density, controllable angle and thickness can be made. The main part of this mechanism consists of an adjustable concave-convex composite mould, multifunction silo, drive screw and framework, can be equipped with force measuring ring, can also be used in combination with ordinary testing machine.

\section{Adjustable composite mould}

The forming quality of pressing mechanism depends on mould. Generally speaking, the forming principle of split mould is simple, but which require higher machining quality, and there is poor roundness after the mould closing, it is difficult to guarantee the shape accuracy of cylinder and uniformity of sample. The mould group with rigid connection have fixed concave-convex mould, can not be disassembled freely, it is not easy to control forming quantity, but easy to waste material. So the seamless pipes machined are used to assemble removable mould group this design.

8 cold-drawn steel cylinder $(2 \times 4)$ of inner diameter $50 \mathrm{~mm}$, height $150 \mathrm{~mm}$ are machined as concave mould group, of which, one end has a 15 degrees blade, and the other end with whorl is assemble with rectangular concave mould plate through a set of slotted round nut. The screw holes are drilled at corresponding positions on convex mould plate, and convex module group could be formed once pressing head are installed. When using, in order to form or demould, people need place smaller round pie into concave mould cylinder as movable pressing head. According to the requirement of different test project, the number of standard sample made each batch could be controlled to $2,4,6$ or 8 . In addition, all components of this mechanism could be disassembled, cleaned, moved and carried easily.

In practice, due to different adhesion and solidification time of different simulation materials, the surface of sample has certain damage during demoulding, so before the material into the mould, people could daub some mold-release agent on the inner surface of concave mould cylinder, such as paraffin wax, silicone oil and graphite etc, to ensure the final molding quality.

\section{Multi-function trough}

Because the molding method this design require the molds are pressed into the material, therefore, as far sample with bedding, before the material into molds, worker need set thickness, inclination, position, number of each layer (according to predetermined design, finished in the trough), which means in addition to holding material, the trough space should be adjustable, should have observation window, and could bear certain expansion pressure.

Eventually, trough of the designed by a side plate, a bottom plate and a movable partition board, which baffle according to selection of molding quantity to separating of the feed slot space, control material usage, material saving. Length direction of the side of the side plate reserved installation position, layered loading, fixed thick organic glass, as the observation window, both real-time observation and material clearing export function. As Fig. 2.

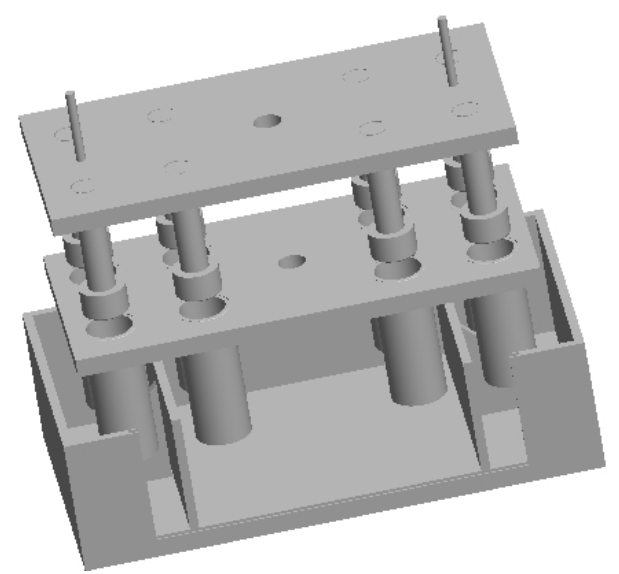

Figure 2. Adjustable composite mould and multi-function silo.

\section{Drive screw and frame}

The design is different from other existing schemes on molding process, therefore, it is necessary to guarantee a certain rolling force by mechanical means. According to the design, in molding process, different pressure value is needed according to different conditions, the maximum value in the convex die compaction link, a single test sample's maximum pressure molding is designed to $3 \mathrm{kN}$, then at the maximum efficiency, the maximum load of pressing 8 samples is $24 \mathrm{kN}$; in loading ways, by rotating the handle, drives the single screw propulsion thrust to produce thrust, converting to linear thrust formulas from rotating torque. As Eq. 1. 


$$
\left\{\begin{array}{c}
P=8 P_{i} \\
T=F d \\
P=\frac{2 \pi \eta_{1} T}{l}
\end{array}\right.
$$

wherein, $P$, the thrust generated by the screw rod; $P_{i}$, the single test piece pressed strength; $T$, the driving torque; $l$, the feed screw guide path; $\eta_{1}$, the positive efficiency of the feed screw; $F$, the handle thrust; $d$, the handle length.

The straight line thrust designed meets the design requirements and proves manual drive mode is feasible. When the bigger compaction force needed, it could be obtained by several ways like (1) connecting a longer handle; (2) reducing each batch forming quantity (3) using press machine loading through conversion joint (4) changing manual drive to motor drive.

In addition, regarding the central screw bearing main pressure, its size design requires for the stability. Taking into consideration of the overall comfort and reserve force measuring ring space, screw length is set as 550 $\mathrm{mm}$, the end part and the frame are connected by sleeve threads, regarded as articulated. The stability safety factor of the design is $n_{\mathrm{st}}=3$, by a slender rod stability formula. As Eq. 2.

$$
\left\{\begin{array}{c}
\frac{P_{c r}}{P} \geq n_{s t} \\
P_{c r}=\frac{\pi^{2} E I}{(\mu l)^{2}} \\
I=\frac{\pi D^{4}}{64}
\end{array}\right.
$$

To reach the conclusion, under maximum screw load, selecting steel as material, the diameter should be bigger than $22 \mathrm{~mm}$, comprehensive consideration of service life, space interference, lead and other issues, a 8.8 grade M27 screw is chosen finally.

The machine frame adopts four column type, connected by two layers beam clapboards, takes into account of the stability problem on material selecting and cross section size designing issues, here no repeat any more.

To quantitative control the pressure, try to ensure the compactness and uniformity of each batch, before each pressure, force measuring ring is placed between central screw and die plate, to get real-time observation pressure data; instead, or the whole mold after loading could be put pressure under pressure machine to get data through online software.

\section{Forming process of the equipment}

The mechanism has the advantages of simple structure and convenient operation, the whole process could be completed by a single person, and the specific forming process consists of 4 steps:

(a) Material compaction. Setting watch window glass, fixing the position of trough, the material which are mixed well according to a predetermined ratio and stirred well are put into trough successively, observing in real time by lateral organic glass window, making different angles and beddings as needed; rotating the handle, letting central screw contact temporary plate to compact material in batches.

(b) Concave mold pressing. Assembling concave mold combination, adjusting to the horizontal plane and tighten the screw in turn, apply mold releasing agent, overall combination is placed on top of the materials, installing vertical positioning guide rod, rotating the handle, pressing concave mold into the materials until contacting anti-sticky pad.

(c) Convex mold compaction. Putting active press plate into concave mold combination successively; assembling convex mold combination, adjusting to the horizontal plane and tighten the screw in turn, positioned on the top of concave mold combination, installing positioning guide rod; putting convex mold into concave mold at a predetermined pressure, re-compacting materials, standing its shaping until primary solidification, removing the watching window glass, opening the trough from the side, cleaning up the scraps outside the mold, eliminating the adhesion with sample, reducing the next step enhancing resistance, keeping static state.

(d) Demolding and forming. After materials coagulation, lifting concave mold mechanism away from $t$ trough by the handle, ascending to a certain height then fixed; reinstalling convex mold combination by single mold release plan and fixing position by guide rod; dropping the central guide rod, putting convex mold into concave mold at a certain speed, demolding the samples. To avoid sample edge damages caused by falling after demolding, proper amount of sand could be put in the bottom of trough as buffer. To prevent corrosion, disassembling and cleaning the parts sticky with materials timely after each forming, brushing oil for maintenance when stopping use for a long time. Complete forming process is referred as Fig. 3.

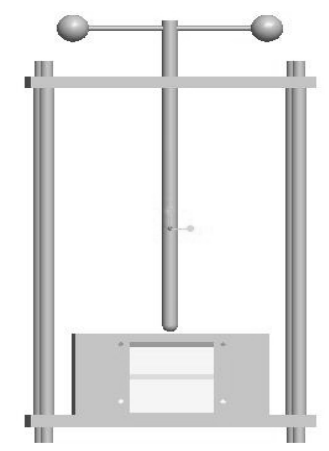

(a) Material compaction

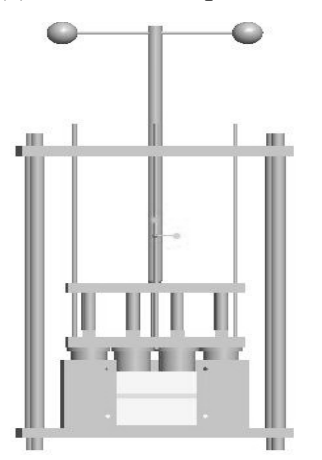

(c) Convex mold compaction

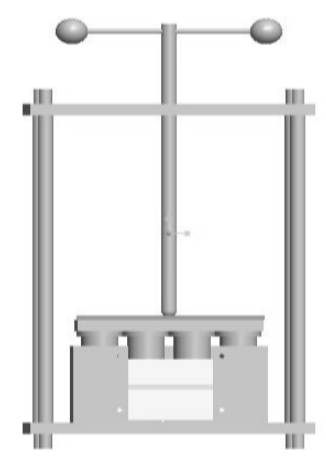

(b) Concave mold pressing

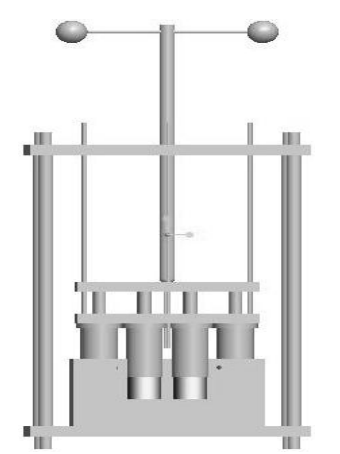

(d) Demolding and forming
Figure 3. Bedded standard samples forming process. 
Finally, the physical mechanism and bedded standard samples made by this mechanism are shown in Fig. 4, the mold quality achieves technical requirements. When putting materials, artificial control could be given to each layer for angle, thickness, and position; the quantity of one time forming could be controlled when installing mold combination; pressing force and sample height could be controlled in compaction process, which has outstanding advantages and is better than other type forming mechanisms for bedded samples making. The design got "four column type geotechnical material batch pressing mechanism for bedded standard samples" [5] and "geotechnical experiment standard samples forming combination mold" [6] two national new practical patents authorization.
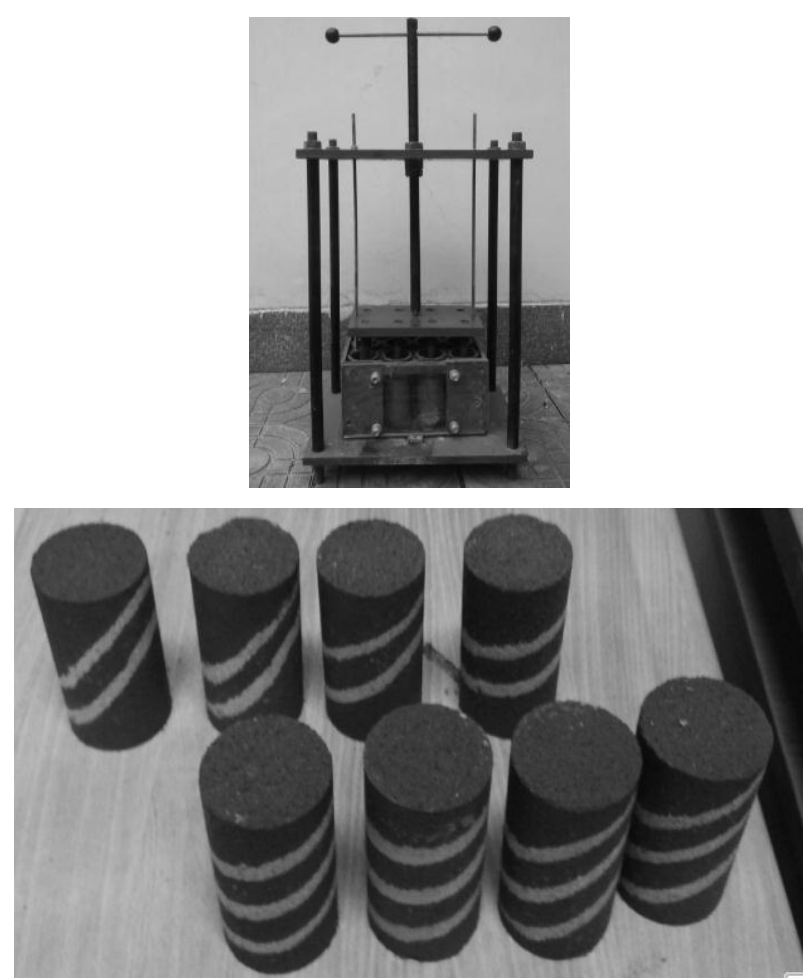

Figure 4. Physical mechanism and bedded standard samples.

\section{Conclusion}

A batch pressing mechanism for bedded standard samples is designed by research on existing standard samples forming mechanisms, by putting mold into materials, each layered material, dip angle, thickness and position can be controlled, forming quantity for each lot is adjustable, uniformity of the same lot is also good and all parts are detachable. The mechanism is suitable for small batch forming and has obvious advantages in bedding controlling, forming efficiency, forming quality and operation convenience, etc. which could be used well in similar material simulation experiment, geotechnical experiment, also could be used to make micro physical models with engineering structure.

\section{Acknowledgement}

I gratefully acknowledge the financial support from the National Natural Science Fund project (51374134), the Chinese Education Ministry doctoral program project (20133719110005), and the Natural Science Foundation of Shandong Province (ZR2014EL20).

\section{References}

1. Z. G. Ma, Study of Simulating Creep Properties of Soft Rock with Latex Cement. Qingdao: Qingdao University of Science and Technology, (2014):43-48.

2. D. Y. Jiang, T. Ren, J. Chen et al. Experimental study of mechanical characteristic of molded salt rock with weak interlayer. Chinese Journal of Rock Mechanics and Engineering, (2012), 31, 9: $1797-$ 1803.

3. Y. M. Tien, M.C. Kuo, C. H. Juang. An experimental investigation of the failure mechanism of simulated transversely isotropic rocks. International Journal of Rock Mechanics \& Mining Sciences. 43 (2006) 11631181.

4. G. M. Zhang, Y. P. Li, X. L. Shi, et al. Research on a model material preparation method for alternate layered rock mass and preliminary experiment. Rock and Soil Mechanics, 2011, 32 (supp.2): 284-289.

5. J. G. Li, Y. Y. Wang, W. X. Wang et al. Four column types batch pressing mechanism for geotechnical materials bedded standard samples: China, 201420140161.6 (P). 2014-07-30.

6. Y. Y. Wang, J. G. Li, W. X. Wang et al. Composite mould for geotechnical test standard sample forming: China, 201420140748.7 (P). 2014-07-30. 